\title{
Microstructure Characterization of Ion-irradiated Ferritic/Martensitic HT9 Steel
}

\author{
Djamel Kaoumi ${ }^{1}$, Ce Zheng $^{1}$
}

${ }^{1}$ Department of Nuclear Engineering, North Carolina State University, Raleigh, 27607, NC, USA

HT9 is a $12 \mathrm{Cr}$ Ferritic/Martensitic (F/M) steel considered as a promising candidate for structural and cladding applications in Generation IV reactors [1]. The chemical composition of the alloy is given in Table 1. The harsh service conditions in Gen IV reactors require that the microstructural response to irradiation of the candidate structural alloys be investigated and understood to qualify them. For that matter, a series of ion irradiations were done. Bulk HT9 specimens were irradiated using $5 \mathrm{MeV} \mathrm{Fe}^{++}$ ions to 20 displacements per atom (dpa) at $600 \mathrm{~nm}$ depth with a dose rate approximately to $5 \times 10^{-4} \mathrm{dpa} / \mathrm{s}$, at irradiation temperatures of 420,440 and $470^{\circ} \mathrm{C}$ (with a variation of $\pm 5^{\circ} \mathrm{C}$ ). The temperature was monitored using an infrared camera and four attached Type $\mathrm{J}$ thermocouples. For post-irradiation characterization, TEM specimens were firstly prepared by the FIB lift-out method using a FEI Quanta focused ion beam (FIB) instrument. ChemiSTEM characterizations were then conducted on FIB laminas using a FEI Titan 80-300 probe aberration corrected microscope. ChemiSTEM characterization was also conducted on as-received HT9 prior to ion irradiation. Only pre-existed $\mathrm{M}_{23} \mathrm{C}_{6}$ type carbides and V-rich nitride precipitates were observed in the as-received condition. In contrast, $\mathrm{Ni}-\mathrm{Si}-\mathrm{Mn}$ rich precipitates (also known as G phase) were found in HT9 irradiated to 20 dpa at 420,440 and $470^{\circ} \mathrm{C}$, as shown in Figure 1. Radiation-induced Ni segregation was also observed at grain boundaries, which is highlighted by white arrows in Figure 2. In addition, the $G$ phase precipitates were found to nucleate heterogeneously along lath grain boundaries, as indexed by red arrows in Figure 2 . The observed results indicated that, under self-ion irradiation, alloying elements such as $\mathrm{Ni}, \mathrm{Si}$ and $\mathrm{Mn}$ segregate at defect sinks, which become thus favourable nucleation sites and promote the radiation-induced $G$ phase precipitation. While radiation induced precipitation and segregation in neutron irradiated F/M HT9 have been widely reported in the literature, similar investigations under ion irradiation have been more scarce $[2,3,4]$. In fact, this study serves to generate baseline data on ion irradiation effects on F/M HT9 in an effort to learn how to more accurately choose ion-irradiation experimental conditions to emulate the irradiated microstructures and effects observed under neutron irradiation.

Ion irradiations were also carried out in the same alloy at similar temperatures in-situ in a TEM using $1 \mathrm{MeV} \mathrm{Kr++}$ ions so that the microstructure characterized in-situ in the TEM can be compared with the microstructure achieved on the same alloys using self-ion irradiation on bulk samples. The focus of the comparison is put on the size and density of dislocation loops induced by irradiation, as well as dislocation loop burgers vector determination. The in-situ experiments provide data on the kinetics of irradiation induced defect formation and evolution, and on the damage spatial correlation with the preexisting microstructure, and thus can help understand how the microstructures observed ex-situ in the bulk samples have developed for, in these latter cases, only snapshots are available at the limited doses. By comparing the ex-situ and in-situ irradiation it is also possible to substantiate the free surface effect on the radiation induced microstructure. The presentation will also report such comparison.

References:

[1] R. L. Klueh, A. T. Nelson, Journal of Nuclear Materials, 371 (2007) p. 37

[2] J. J. Kai, G. L. Kulcinshi, Journal of Nuclear Materials, 175 (1990) p. 227

[3] E. Getto et al, Journal of Nuclear Materials, 480 (2016) p. 159

[4] E. Getto et al, Journal of Nuclear Materials, 484 (2017) p.193 


\begin{tabular}{|l|l|l|l|l|l|l|l|l|l|l|l|}
\hline $\mathbf{F e}$ & $\mathbf{C r}$ & $\mathbf{M o}$ & $\mathbf{N i}$ & $\mathbf{M n}$ & $\mathbf{W}$ & $\mathbf{V}$ & $\mathbf{S i}$ & $\mathbf{C}$ & $\mathbf{N}$ & $\mathbf{P}$ & $\mathbf{S}$ \\
\hline 84.89 & 11.8 & 1.03 & 0.51 & 0.5 & 0.5 & 0.33 & 0.21 & 0.21 & 0.01 & 0.008 & 0.003 \\
\hline
\end{tabular}

Table 1. As-received HT9 chemical composition (wt.\%)

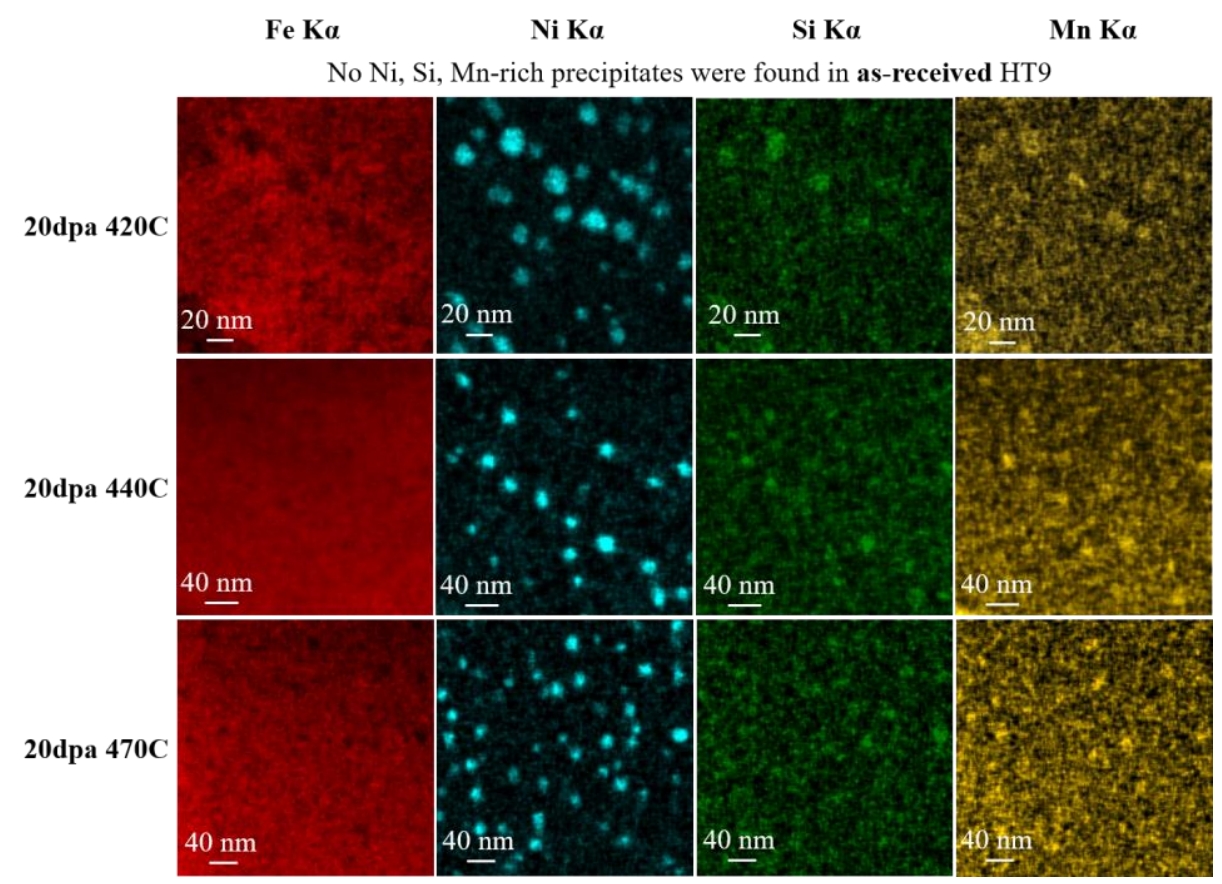

Figure 1. ChemiSTEM maps showed $\mathrm{Ni}, \mathrm{Si}$ and $\mathrm{Mn}$ enrichment (associated with Fe depletion) in HT9 self-ion irradiated to $20 \mathrm{dpa}$ at 420,440 and $470^{\circ} \mathrm{C}$. It indicates the formation of $\mathrm{Ni}, \mathrm{Si}, \mathrm{Mn}$-rich precipitates in HT9 under irradiation.

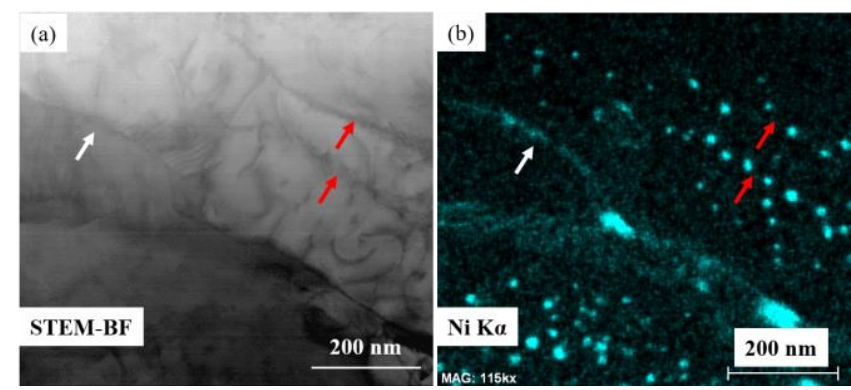

Figure 2. STEM mode bright field micrograph (a) and corresponding Ni Ka map (b) of HT9 self-ion irradiated to $20 \mathrm{dpa}$ at $440^{\circ} \mathrm{C}$; Ni segregated to grain boundaries (indexed by white arrows) and some of $\mathrm{Ni}, \mathrm{Si}, \mathrm{Mn}$-rich precipitates nucleated heterogeneously along lath grain boundaries (indexed by red arrows). 